\title{
Impacto de los recursos digitales abiertos en el aprendizaje de la estadística en el nivel medio superior
}

Impact of open digital resources on the learning of statistics at the upper secondary level

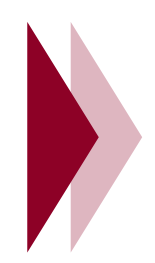

Jesús Mario Zorrilla

Ingeniero industrial, con Diploma en Evaluación por competencias; magister en Educación con énfasis en Procesos de Enseñanza-Aprendizaje. Docente CAU Cali - Universidad Santo Tomás.

Correo electrónico: jesuszorrilla@ustadistancia.edu.co 


\section{Resumen}

La presente investigación es de orden cualitativo y tiene como propósito fundamental medir el impacto que tiene la utilización de recursos educativos abiertos en el aprendizaje de la Estadística en el nivel medio superior dentro de una institución educativa que aplica un modelo pedagógico no tradicional, y en la que la utilización de dispositivos de tipo tecnológico está delimitada por los propios fundamentos de la pedagogía Waldorf. Para llevar a cabo este proceso, se parte de la pregunta de investigación: ¿De qué manera influye la utilización de recursos digitales educativos abiertos en el aprendizaje para estudiantes del grado noveno de educación media del colegio Waldorf Luis Horacio Gómez de Cali-Colombia? Para responder a este interrogante, se utilizaron recursos disponibles en la red de Internet para ser consultados por los estudiantes, y posteriormente se emplearon cuestionarios, entrevistas y registros de observación, con los cuales, al entrar en el proceso de análisis de los datos, se obtuvieron hallazgos que fueron categorizados, y así llegar a las conclusiones del estudio, junto con recomendaciones y sugerencias para próximos trabajos que involucren los recursos disponibles en la red para el ámbito educativo.

Palabras clave: recursos educativos, abiertos, estadística, pedagogía Waldorf.

\section{Abstract}

The present research is of a qualitative nature and its main purpose is to measure the impact that the use of open educational resources has on the learning of Statistics at the upper secondary level within an educational institution that applies a non-traditional pedagogical model, and in the that the use of technological devices is limited by the very foundations of the Waldorf pedagogy. To carry out this process, we start with the research question: How does the use of open educational digital resources affect learning for students in the ninth grade of middle school education at Waldorf Luis Horacio Gómez School in Cali, Colombia? To answer this question, available resources were used in the Internet to be consulted by the students, and later questionnaires, interviews and observation records were used, with which, when entering the process of data analysis, obtained findings that were categorized, and so reach the conclusions of the study, along with recommendations and suggestions for future work involving the resources available in the network for the educational field.

Keywords: educational resources, open, statistics, waldorf pedagogy.

\section{Introducción}

El colegio Waldorf Luis Horacio Gómez, en el cual se lleva a cabo la presente investigación, es una institución de carácter privado, ubicado en la ciudad de Cali- Colombia, en la zona perimetral al sur de la ciudad, entidad que ha estado funcionando desde hace 40 años. La institución cuenta con tres niveles de enseñanza: preescolar, primaria y media básica, en los que existe un grupo por cada nivel, hasta llegar al grado undécimo $\left(11^{\circ}\right.$ grado), de acuerdo con las disposiciones emanadas del Ministerio de Educación Nacional de Colombia (M.E.N.).

El horario de clases está diseñado con base en el fundamento antropológico que enmarca el modelo de la pedagogía Waldorf; en todos los niveles de escolaridad, de siete a nueve de la mañana se desarrolla lo que se llama la clase principal, la cual tiene recurrencia en épocas de tres a cuatro semanas, ya que este espacio representa el mejor 
momento para el aprendizaje, por cuanto los estudiantes se encuentran con mejor disposición para lo cognitivo; luego viene un descanso de media hora, y se continúa con la llamada clase de refuerzo, que también se ajusta a las épocas contempladas en la clase principal.

En este orden de ideas, el área de las matemáticas también se encuentra enmarcada dentro de los lineamientos y directrices determinados por el fundamento pedagógico aplicado, entendiendo que a cada etapa evolutiva del niño se asocian distintas necesidades que coadyuven en su desarro1lo, y es por esta razón que no se permite el uso de dispositivos electrónicos dentro del colegio, y no se fomenta la aplicación de la tecnología hasta los dos últimos grados de escolaridad; es decir, en los otros grados escolares distintos al grado décimo y undécimo, ni siquiera es permitida el acceso o la consulta de tareas e investigaciones en sitios con contenidos virtuales.

Al indagar acerca de investigaciones pedagógicas relacionadas con el tema de los recursos tecnológicos abiertos orientados hacia el campo de la investigación educativa, se halla que hay algunos trabajos en los cuales los resultados encontrados permiten entregar productos auspiciosos (Ferro, 2009). Es así como se vislumbra que el uso e implementación de recursos educativos abiertos enriquecen las clases, en la medida en que haya una planeación eficaz de las mismas, dando la oportunidad al docente de identificar el modelo de enseñanza que debe implementar; así mismo, se puede encontrar que es posible desarrollar competencias matemáticas en la resolución de problemas de manera autónoma, interactuando de manera activa y por sus propios medios (Cervantes, 2012); pero es importante destacar igualmente que en los trabajos realizados se plantean líneas de acción que pueden seguirse en futuros trabajos, como la profundización en la investigación de los propios alumnos como diseñadores de actividades (Rendón, 2012), ya que ellas representan formulaciones interesantes en la innovación de las prácticas educativas diseñadas a partir de la utilización de los recursos digitales (Área, 2008).

\section{Antecedentes del problema}

Contrariamente a la opinión generalizada por muchos docentes, acerca de que existe un aprendizaje preferido en las personas, se tiene la convicción de que el ser humano posee distintos modos de aprender, en diferentes contextos y situaciones, con ritmos variados, y en todos los momentos de su vida, de acuerdo con sus diferentes experiencias, actitudes y talentos (Mc Geal, 2011). Dentro de este contexto, en las tres últimas décadas se ha venido trabajando en la teoría de las inteligencias múltiples, modelo propuesto por el psicólogo e investigador norteamericano Howard Gardner, según el cual todos los seres humanos poseen ocho inteligencias, las cuales se combinan de diversas formas para adquirir finalmente el aprendizaje (Gardner, 1995); posteriormente, en la última década del siglo anterior, el también psicólogo estadounidense Daniel Goleman, incorporó el término inteligencia emocional como un componente adicional que también puede influir en el intelecto y que puede aportar posibilidades amplias para el desarrollo integral de las personas (Goleman, 1997).

Observando que existen gran cantidad de factores que logran tener un impacto significativo en los estilos de aprendizaje, y que en determinados procesos educativos los estudiantes demuestran inclinación hacia ciertos estilos pedagógicos, es preciso dar una mirada a la forma en la cual los avances tecnológicos de la información y las comunicaciones han incursionado en el contexto educativo, y de qué manera todo este conjunto de productos y herramientas están impactando la diversas dimensiones de la vida escolar actual.

Al integrar y desarrollar nuevas propuestas del campo tecnológico relacionadas con la educación, es fundamental considerar que el acceso a los recursos educativos abiertos y su posterior utilización no representa costo alguno para los usuarios, ya que son promovidos por centros educativos, instituciones universitarias y organizaciones dedicadas al fomento de la investigación y la capacitación, logrando que tanto estudiantes como 
docentes y/o personas interesadas en el continuo proceso de aprendizaje sean los directos beneficiarios de esta oferta de posibilidades.

En nuestro contexto local, resulta vital la forma en la cual se aborda la investigación, ya que el fundamento pedagógico, como se indicó anteriormente, es muy categórico en señalar que la utilización de los medios tecnológicos y la exposición al uso en exceso afectan el normal desarrollo de los niños y por lo tanto no se debe promover el acceso a estos dispositivos electrónicos. Es muy difícil llegar a determinar que tanto se puede afectar el aspecto antropológico cuando se accede al uso de estos medios, pero si es verdad que se puede llegar a establecer que tanto se puede mejorar la adquisición del conocimiento al utilizar recursos educativos abiertos: ese es el gran reto del presente trabajo de investigación.

\section{Planteamiento del problema}

Este trabajo pretende responder y aportar información válida a la comunidad educativa en general, a partir de la pregunta: ¿De qué manera influye la utilización de recursos digitales educativos abiertos en el aprendizaje de la estadística para estudiantes del grado noveno de educación media del colegio Waldorf Luis Horacio Gómez de Cali-Colombia? El interrogante planteado busca relacionar dos variables: 1) la utilización de recursos abiertos disponibles en la red de información y 2) su influencia en el aprendizaje y adquisición del conocimiento por parte de los estudiantes.

Las evaluaciones realizadas a estudiantes indican que a pesar de haber recibido sus grados de escolaridad y acreditarlos debidamente, acceden al contexto laboral con niveles muy bajos de competencias, según registro del Observatorio Laboral de México en su boletín informativo con última modificación en marzo del 2014, existiendo un riesgo muy grande de realizar su ejercicio profesional de manera deficiente; es por ello que desde la escuela se deben incluir procesos e investigaciones en los cuales se incremente en forma gradual los niveles de complejidad, hasta llegar a alcanzar una competencia específica con mayor grado de detalle. Para ello hay que motivar a los estudiantes a que se interesen por el conocimiento, por lo cual surge una pregunta subordinada a la pregunta principal, la que también será respondida en el curso de la investigación, y es la siguiente: ¿Qué grado de motivación se ha manifestado en los alumnos para hacer uso de los recursos educativos tecnológicos?

\section{Objetivos de la investigación}

- Analizar la incidencia de la utilización de recursos educativos tecnológicos abiertos en el aprendizaje de contenidos en la estadística.

- Evidenciar la utilización de la tecnología como recurso pedagógico y herramienta de apoyo en el aprendizaje, aprovechando las ventajas que representan, de fácil comprensión, flexibilidad y con facilidad para el acceso de la comunidad estudiantil.

- Evidenciar el aprendizaje de los estudiantes con propuestas dinámicas y atractivas, en donde sean motivados para profundizar en la investigación científica, a partir de temas desarrollados con los recursos de la tecnología actual, promoviendo novedosas formas de evaluación.

\section{Limitaciones de la investigación}

La investigación encuentra limitaciones inicialmente en cuanto a que en la institución no existe una sala multimedia en donde se pueda disponer en forma organizada a los estudiantes y en la que se tenga la conexión a la red de internet; se trata entonces de dar orientación acerca de cómo deben acceder al uso de los recursos tecnológicos y entregar pautas muy precisas para saber cuál es el tipo de trabajo e investigación que se espera puedan realizar. 
En cuanto a la población estudiantil en la que se realiza la investigación, se pueden encontrar limitaciones en el sentido que existe un solo grado noveno y por lo tanto no se puede aplicar un factor que permita comparar cuantitativamente con otro grupo de estudiantes, y poder determinar así el grado de incidencia de la utilización del recurso educativo abierto entre dos poblaciones. La medición final se puede establecer con base en una evaluación que permita determinar que tanto se alcanza el objetivo planteado; quedará para trabajos posteriores el poder cuantificar la diferencia entre los dos estilos: el que tradicionalmente se ha aplicado en el aula, y el que se hacer con la utilización de R.E.A.

\section{Aspectos pedagógicos en la enseñanza de las matemáticas}

La enseñanza de las matemáticas siempre ha hecho parte de las distintas miradas que históricamente se han acercado a explicar la didáctica educativa, por lo cual se podría hablar de dos corrientes claramente establecidas. Existe una corriente enmarcada como mecanicista, correspondiente a aquellos docentes que hacen de la labor pedagógica una tarea enmarcada en la transmisión de un conocimiento finalizado y abstracto, en la que predomina una tendencia a adoptar un estilo expositivo (Godino, 2003). Aquí, la transmisión del conocimiento se llena de definiciones en abstracto, y de procedimientos algorítmicos, dejando lo que corresponde a la resolución de problemas para una parte final en la que se plantea un taller de ejercicios (Breci, 2012); de cierta forma, esta perspectiva educativa lo que hace es convertir el conocimiento matemático en algo trivial, rutinario, que es totalmente controlado y controlable por el docente.

Contrariamente a esta línea, existe una consideración en la que el conocimiento matemático no puede asumirse como totalmente finalizado, sino que es algo en constante creación, que es algo más que definiciones entregadas para ser memorizadas, donde las estructuras correspondientes a los conceptos se enriquecen y aumentan cada día, en donde ya no será suficiente con lo magistral de la clase y su rigor: debe hacerse que el alumno se involucre y construya su propio conocimiento; y la forma de integrarlo y hacerlo partícipe es simplemente tratar de que todo lo que se enseñe tenga un significado válido; esto hace parte de la corriente constructivista del aprendizaje (Herrington, 2000).

En el proceso de desarrollar los hábitos de pensamiento matemático, debe vincularse a los alumnos para que participen en la construcción del conocimiento, siendo esto tan fundamental como el mismo hecho de exponerlo. Sin embargo, es interesante ver como estos aportes han logrado enfocar a los alumnos en una actividad que ayuda a la construcción del conocimiento, conocida como resolución de problemas; a través de esta técnica, se pretende transmitir sistemáticamente los procesos de pensamiento que se consideren útiles y eficaces para resolver verdaderos problemas (De Guzmán, 2007). Se podrá entonces ejercitar la creatividad del alumno, activar su capacidad mental de una manera divertida, entregándole la confianza necesaria para abordar situaciones que se presentan en su cotidianeidad. Esta estrategia puede ofrecer un campo fértil y óptimo para el aprendizaje, siempre y cuando exista una planificación adecuada en cuanto a las situaciones planteadas que se ejecutan al interior del aula.

Más allá de los distintos modelos en que se pueda fundamentar la didáctica de las matemáticas, el hecho mismo de desarrollar en los estudiantes competencias para la vida, obliga a replantear estrategias que utilicen conceptos contemplados en modelos aparentemente opuestos. Por ejemplo, en la propuesta del aprendizaje significativo de David Ausubel, se distinguen tres tipos de aprendizajes:

- Aprendizaje de representaciones: atribuye significado a símbolos (verbales o escritos) a través de la asociación de éstos con sus referentes objetivos.

- Aprendizaje de conceptos: abstrae de la realidad atributos comunes a los objetos que les hace pertenecer a determinada categoría. 
- Aprendizaje de proposiciones: asimila el significado de términos o símbolos aislados producto de una combinación lógica de términos.

Así mismo, el hecho de dar relevancia al aprendizaje por descubrimiento de contenidos no representa la negación del aprendizaje expositivo, pues en algún momento, este último puede ser igualmente eficaz (Tapia, 1995).

La educación hoy en día ha puesto su mirada en desarrollar competencias, y en este sentido los alumnos deben estar en capacidad de lograr la conceptualización y de llegar a la resolución de situaciones; el modelo holístico de enseñanza se constituye en un elemento aplicativo que puede facilitar el desarrollo y alcance de los objetivos de la enseñanza a partir de los contenidos curriculares, ya que los diferentes saberes: el saber que, el saber cómo, el saber donde, el saber cuándo, el saber porque, el saber para qué y el poder saber, se contienen mutuamente dentro de su propia estructura. Esta visión educativa señala en esencia que existen cuatro tipos de aprendizaje necesarios en las nuevas comunidades escolares del siglo XXI (Fernández, 2006), los cuales se muestran a continuación:

- Aprender a aprender: se refiere a la capacidad para proseguir y persistir en el aprendizaje, es decir, organizar el propio aprendizaje.

- Aprender a hacer: se refiere a la ejecución de procedimientos y estrategias, técnicas y habilidades, destrezas y métodos.

- Aprender a ser: se refiere al reconocimiento del otro como persona, con todas sus implicaciones, como lo son el reconocimiento de su libertad y el respeto a todos sus derechos.

- Aprender a vivir juntos: se refiere a aprender a conocer y respetar al otro, a lograr acuerdos, a encontrar soluciones por consenso.

Con la aplicación de los criterios de esta estrategia de enseñanza, se puede llegar a la formula- ción de planes de mejoramiento individuales que conduzcan a mejorar el nivel de logro de todos los alumnos.

\section{Estrategias de evaluación}

Con el cambio en las políticas educativas surgidas en el último tiempo, especialmente en los países en vía de desarrollo, se ha generado todo un movimiento que busca replantear los paradigmas en el concepto de educación. Sin embargo, al interior de las aulas de clase, ellas no han sido plasmadas en forma contundente y efectiva, especialmente al momento de evaluar al alumno. Al proponer una evaluación real y efectiva, que reivindique los procesos desarrollados para la adquisición del conocimiento, hay que considerar que esta evaluación se encuentre en concordancia con el planteamiento curricular, con los desarrollos dentro del aula, las metodologías utilizadas y los niveles a evaluar.

La evaluación no solamente exige que se indague acerca del conocimiento final, sino que se debe hacer un proceso de observación para saber si el estudiante se encuentra en condiciones de plantear y resolver problemas; para ello se deben recrear situaciones en las cuales se favorezcan los procesos investigativos y de indagación. Ahora, cuando corresponde realizar una evaluación por competencias, "se puede apreciar con facilidad que el proceso de evaluación se complejiza, ya que es muy fácil perder de vista lo que se quiere medir y sobre lo que hay que determinar un juicio valorativo" (Lozano, 2013).

La evaluación va a tomar en cuenta entonces, el desarrollo de las competencias determinadas para cada uno de los campos contemplados en el desarrollo curricular, y que solamente se pueden cualificar si se considera los procesos de pensamiento, que son en últimas, las competencias básicas planteadas dentro del lineamiento cognitivo; son ellas quienes van a consolidar los insumos que permitan procesar la información, no desde 
el método memorístico como tal, sino con el propósito que favorecer las estructuras enmarcadas en la resolución de problemas, o sea, su aplicación funcional en el quehacer diario.

\section{El aprendizaje apoyado en la tecnología}

El aprendizaje a través de medios electrónicos, es una herramienta que se ha venido incrementando ampliamente, en la medida del avance de la tecnología misma, pero el mayor auge se ha dado en los últimos años, a partir de las experiencias en aquellos países en los cuales las telecomunicaciones y la sociedad de la información adquirieron un mayor desarrollo e impacto social en este mismo tiempo (Quintanilla, 1991).

Tal auge y desarrollo de esta modalidad de aprendizaje apoyada en medios tecnológicos, se ha contextualizado por medio del concepto de e-learning, simplificación del término "electronic learning" , y que hace referencia a la modalidad de enseñanza-aprendizaje que consiste en el diseño, implementación y evaluación de un plan de formación y/o capacitación desarrollado a través de ordenadores y el cual es ofrecido a personas de variadas ubicaciones geográficas, dentro de un entorno virtual (Área, 2008).

El e-learning se ha transformado de a poco en un aprendizaje emergente, conformado por un sistema auto-organizado afectado por los contextos actuales del aprendizaje y bajo la influencia de la red de información; es un sistema que propicia el aprendizaje a través del fomento de la experimentación y la innovación, utilizando la flexibilidad que se genera a partir de los espacios de interacción que se generan. En esta modalidad de aprendizaje emergente se exploran las combinaciones provenientes de un aprendizaje informal, con todos los recursos tecnológicos que proliferan en la red: portales dinámicos, blogs, redes sociales, chats, mensajería instantánea, storytelling, know how pedagógicos, a los que se les agrega la autogestión del conocimiento, la colaboración dentro del ámbito y otros (Stephenson, 2001).

Dentro de las ventajas que puede ofrecer esta metodología del aprendizaje, se cuenta con la inmediatez del acceso a la información, con gran variedad de perspectivas y puntos de vista, en contextos socioculturales variados, sin barreras demográficas o geográficas, lo cual enriquece el proceso del conocimiento; estos ítems permiten el desarrollo de habilidades para el manejo de herramientas de diversa índole, generando experiencias llenas de matices, por lo cual se puede alcanzar un aprendizaje prácticamente autodidacta, pues aunque hay guías en diferentes partes del proceso, existe la autonomía para la profundización de temas y contenidos. Esta forma de aprendizaje, ha permitido que mayor cantidad de personas se interesen por mejorar su nivel académico, ya que muchas de ellas no tenían la posibilidad de acceso a los métodos convencionales de escolarización, siendo éste un sistema que brinda la posibilidad de alternar trabajo y estudio, a un bajo costo, con rapidez en la comunicación, y con posibilidades de interacción continua con personas en condiciones similares de aprendizaje.

Dentro de los inconvenientes que se pueden mencionar, hay que considerar que es necesario contar con unas mínimas competencias en el campo de la tecnología, lo cual restringe el acceso a los contenidos en determinadas circunstancias; igualmente se deben desarrollar habilidades para el aprendizaje autodidacta, autónomo y colaborativo, de las cuales hay carencia en muchas personas; en igual forma, para los docentes siempre se requiere una mayor dedicación de tiempo profesional, especialmente en la parte inicial del proceso.

Para contextualizar la importancia del e-learning se hace necesario comprender los dos componentes del sistema: el componente pedagógico que hace relación a la tecnología educativa como disciplinada integrada a la tecnología, la psicología y la didáctica, y los cuales van a trabajar directamente sobre los contenidos, convirtiéndose 
en el alma del aprendizaje; y el componente tecnológico, que contiene todo lo referente al diseño, selección, implementación y mantenimiento de soluciones informáticas de aplicación abierta sin restricción por derechos de autoría, y los cuales permiten la administración y control de la capacitación como tal.

Con la consolidación del e-learning, aparecen también propuestas similares, que se conocen como el b-learnig (blended-learning), un concepto asociado a la modalidad de aprendizaje semipresencial, y el h-learnig (home-learning), que se relaciona con el aprendizaje a través de una extensión de tiempo que complementa el contenido esencial de los temas en desarrollo (Heinze, 2004). De una parte, la apertura de programas, contenidos y prácticas tiene ventajas considerables, mencionadas anteriormente, para todos los ámbitos escolares, ya que es una forma de potencializar el acceso a la educación, lo cual genera equidad social e igualdad en las oportunidades, mejorando el proceso académico; pero por otra parte, no solamente se trata de analizar los distintos modelos pedagógicos y sus contenidos en los cuales se ve inmerso el aprendizaje virtual: debe fomentarse la participación activa, a sabiendas que se trata de un universo cambiante, en el cual se hace imprescindible desarrollar competencias que permitan identificar y desarrollar modelos variados del conocimiento.

A la par del aprendizaje electrónico, se incorpora el concepto de TIC, o tecnologías de información y comunicación, que en el sentido pedagógico hacen alusión a las tecnologías necesarias para la gestión y transformación de la información, y los programas que permiten la transmisión, procesamiento y difusión de la información en todas sus facetas, para optimizar el manejo de los datos y generar mayor conocimiento.

La utilización de las TIC, en los contextos educativos, es cada día más apreciable; es un cambio de realidad, en el cual su incorporación al aula de clase y su consolidación en los procesos de aprendizaje, constituye un desafío dentro de la imple- mentación de una didáctica educativa planteada a partir de los propios maestros, sin afectar la estructura del modelo pedagógico que se sigue. Estas transformaciones deben ser acompañadas de profundos análisis curriculares y de contenidos, espacios que potencialicen el aprendizaje y apertura e innovación hacia los cambios constantes.

\section{Incorporación de la tecnología en la enseñanza de las matemáticas}

El área de las matemáticas, hasta mediados del siglo pasado, solamente propuso ligeros cambios hasta mediados del siglo anterior, hasta que gracias a la figura del matemático alemán Félix Klein (1849-1925), se cambia el panorama educativo, generando una profunda transformación de la enseñanza, especialmente en lo que se refiere a estructuras abstractas, al rigor de la lógica y a la aplicación de la teoría de conjuntos (De Guzmán, 2006).

A partir de finales del siglo $\mathrm{XX}, \mathrm{y}$ con el auge vertiginoso de la tecnología y su incursión en el ámbito educativo, las matemáticas se convirtieron en una de las áreas que mayormente se vieron impactadas, lo cual se observa incluso desde la inserción misma de la calculadora, que logró desplazar el uso de accesorios tradicionalmente empleados para cálculos aritméticos, y que en su momento brindó rapidez y manejo de datos considerables, situación que aún se mantiene, con la incursión y manejo de las calculadoras científicas. Con todo y sus ventajas, es importante considerar que la incorporación de tecnologías al proceso pedagógico, con la validez social y académica que las acompaña, pueden llegar a convertirse en elementos de apoyo para el aprendizaje, ayudando en la integración del estudiante a su contexto educativo. El objetivo básico, entonces, de las herramientas tecnológicas viene a debe ser la facilitación del aprendizaje y la motivación para la adquisición del conocimiento (Artigue, 1995), logrando así la legitimidad educativa desde el orden pedagógico. 
Es recomendable que la inclusión de la tecnología de las matemáticas al aula, se maneje con mucha cautela y precaución, ya que constituye un elemento de apoyo en la solución de la problemática suscitada con el área, pero con la conciencia de que ella está lejos de constituirse en la panacea para la adquisición del conocimiento y la conquista de las competencias dominantes. En su incorporación, hay que tomar en cuenta cuáles son los ámbitos del aprendizaje que le dan significación al conocimiento en construcción, y a partir de ahí, determinar cuáles de las tareas pueden ser apoyadas por dichas tecnologías, de tal modo que se conviertan en material relevante para la búsqueda del conocimiento, y no se queden en simples procesos algorítmicos.

De acuerdo con Miguel de Guzmán, la educación matemática de hoy en día debe estar inmersa en las formas propias del análisis matemático, enfatizando especialmente en la experiencia y en la manipulación de los elementos de donde proviene, para luego apelar al proceso de abstracción, lo cual le permite imprimir un carácter de rigurosidad y formalismo, tan necesarios para llegar al conocimiento. Ello debe conducir al maestro a estimular a sus estudiantes a la búsqueda autónoma, al descubrimiento metódico de estructuras matemáticas simples y de cómo aplicarlas en forma práctica en la resolución de problemas interesantes (De Guzmán, 2006). Es una búsqueda continua que siempre debe ser orientada, de tal modo que se fomente el placer de descubrir, que permita desarrollar estrategias de pensamiento enfocadas en la vivencia individual.

\section{Utilización de Recursos Educativos Abiertos (R.E.A.)}

La presente investigación se enfoca en los elementos que constituyen objeto de aprendizaje, entendiendo a estos como "el conjunto de recursos disponibles, autocontenibles y reutilizables, con un propósito educativo y que se encuentran conformados por tres componentes internos a saber: contenidos, actividades de aprendizaje, y elementos de contextualización" (M.E.N., 2006, p. 16). En este orden de ideas, y en vista de la falta de decisión de los gobiernos por promover cambios en las condiciones de investigación científica y en la generación de conocimientos, se ha visto la necesidad de compartir materiales digitalizados de manera pública y gratuita, de tal modo que puedan ser utilizados en la enseñanza, en el aprendizaje y en los procesos investigativos por parte de los docentes y estudiantes.

La Unesco, dentro de la declaración de sus principios, siempre ha considerado que la posibilidad de brindar acceso a una educación de gran calidad constituye un factor esencial en la construcción de una sociedad justa y en paz; es así, como en el año 2002, dentro del foro de impacto de Open Courseware para la educación superior en países en desarrollo, adoptó el término Recursos Educativos Abiertos (R.E.A.), definiéndolos como aquellos recursos para la enseñanza, el aprendizaje y la investigación, que son de dominio público o que se hayan publicado bajo licencia de propiedad intelectual, de tal modo que esté permitido el uso libre para otras personas (Delours, 1996). El surgimiento de los R.E.A. se genera a partir del desarrollo de los llamados software de código abierto, manteniendo la concepción de que cuando se puede interpretar, utilizar, modificar y distribuir continuamente el código fuente de un programa, este puede avanzar y evolucionar para ser cada vez mejor (Arma,2012).

La utilización de los Recursos Educativos Abiertos en cualquier ámbito del aprendizaje, conlleva a relacionar la forma en que se pueden utilizar dichos recursos, desde la estructura del contenido buscado, y el impacto que este conocimiento logre generar entre los estudiantes (García, 2008); es decir, se pueden plantear dos perspectivas desde su implementación misma:

a) Como debe ser la práctica docente enfocada en la formación y evaluación de competencias en el estudiante. b) Cuál es la influencia de dicha práctica en el desarrollo y rendimiento académico de los estudiantes. 
La utilización de Recursos Educativos Abiertos permite generar nuevas experiencias para la adquisición del aprendizaje, a la vez que otorga flexibilidad en sus procesos, entregando igualdad en oportunidades educativas y de capacitación para distintos ámbitos, con propuestas que se constituyen en alternativas de bajo costo, fomentando el aprendizaje colaborativo y la auto-construcción del aprendizaje. La gran ventaja que presentan los portales que ofrecen REA radica en que sus contenidos facilitan la interacción, ya que ellos son susceptibles a ser compartidos, mezclados, reajustados y mejorados con relación al contenido inicial, lo cual permite su reutilización continua, incluso al ser traducidos a otros idiomas (Serrano, 2010).

En la clasificación de los Recursos Educativos Abiertos, se han distinguido las siguientes categorías: los contenidos educativos, las herramientas y los recursos de implementación:

- Contenidos educativos: como lo indica su nombre, obedece a todo aquel material que proporciona la información necesaria para la asimilación de los temas del conocimiento a alcanzar; se incluyen los cursos completos a desarrollar, el material de apoyo bibliográfico, los módulos de contenido, textos, materiales audiovisuales multimedia, exámenes, recopilaciones, jornales especializados, etc. Proyectos relacionados: Eduteka (español); Cursos del MIT (español); Proyecto Gutenberg (español); Objetos de aprendizaje (Colombia Aprende);Smithsonian Institute; EducaNext; Discovery Channel; Monterrey Institute for Technology; Universidad Virtual (Unesco); Observatorio de contenidos para e-Learning; Youtube/educación; Reseña de repositorios de contenidos educativos.

- Herramientas: están constituidas por los programas tecnológicos de apoyo (software), registros para la organización de contenidos, implementación de redes de aprendizaje, accesos para la gestión y mejoramiento de sistemas de información, diseños de gestiones para la retroalimentación, etc.
Proyectos relacionados: Moodle; Reseña de herramientas y estándares; OpenCourse; EduCommons.

- Recursos de implementación: constituidos por todo aquel material que permite y apoya el acceso a la información de los contenidos educativos, los diseños de plataformas para el trabajo virtual, las licencias de propiedad intelectual que fomentan la publicación abierta y gratuita de material educativo, así como la adaptación y localización de contenidos. Proyectos relacionados: Reseña de licenciamientos; Creative Commons; Iniciación al OpenCourseWare (MIT); Tutoriales sobre REA; Licencia GNU (copyleft); Red de Uso Honrado; Licencia de Contenido Abierto (OPL).

\section{Método de investigación}

La investigación en curso pretende dar respuesta a la pregunta acerca de cómo influye la utilización de Recursos Educativos Abiertos en el aprendizaje de la estadística, para lo cual es preciso utilizar una propuesta metodológica de tipo cualitativo, ya que se trata de un estudio que proporciona descripciones a partir de observaciones realizadas a un grupo de personas, e indagaciones a ellas mismas (Jiménez, 2000), y además, de acuerdo con la definición entregada por Hernández, Fernández y Baptista (2010), entre los objetivos de investigación correspondientes al paradigma cualitativo, está la descripción, comprensión e interpretación de los fenómenos según el significado producido entre los participantes del estudio, elementos todos que se encuentran involucrados en este estudio.

En este orden de ideas, de acuerdo con Hernández, Fernández y Baptista (2010), el enfoque cualitativo utiliza la recolección sin medición alguna de tipo numérico para encontrar o afinar preguntas de investigación, y puede o no probar hipótesis en su proceso de investigación. Al retomar el planteamiento del problema, se encuentra que con el presente estudio se pretende analizar caracterís- 
ticas que subyacen dentro de un contexto escolar natural relacionadas con el aprendizaje en una de las ramas del campo matemático, como lo es la estadística; en este sentido, se busca encontrar una interpretación a los hallazgos derivados del estudio, sin establecer comparaciones con otros grupos de estudiantes y sin llegar a la generalización de los resultados, lo cual reafirma porque en la escogencia del enfoque mencionado.

Seleccionar este método de investigación permitió llegar a los objetivos planteados en el estudio, porque en el investigador existían características de competencia e idoneidad que permitieron informar con objetividad, precisión y claridad acerca de las propias observaciones, así como de las experiencias de quienes intervienen a lo largo del estudio. En los lineamientos de la pedagogía Waldorf se trabaja en forma sistemática los procesos de observación continua y de indagación, lo cual constituye una garantía para el estudio.

Finalmente, hay que resaltar que el método cualitativo es una técnica que permite la interpretación y comprensión del significado que hay en las acciones desarrolladas por los participantes del estudio y cómo ellas contribuyen a la construcción de conocimiento, sin llegar a ejercer algún tipo de manipulación durante el proceso. En este sentido, una gran ventaja para dar respuesta al problema de investigación, radica en la importancia que el método cualitativo le otorga a la realidad tal y como se vive, tratando de comprender la naturaleza profunda de dicha realidad y su estructura (Martínez, 2000).

\section{Instrumentos de recolección de datos}

Como se planteó anteriormente, el proceso de investigación en desarrollo se encuentra encaminado a obtener una mayor comprensión del fenómeno educativo que involucra las variables incidencia en el aprendizaje y recursos educativos abiertos, por lo cual al finalizar el estudio se presentará una explicación a los hallazgos encontra- dos a lo largo del camino. Es en este sentido, que los instrumentos utilizados para la recolección de los datos de la investigación, corresponden esencialmente a los más comunes contemplados dentro del campo de la investigación cualitativa: la observación, la entrevista y los documentos (Flórez, 2011).

Seguidamente, en forma breve se explicarán las razones de su escogencia y el alcance de las mismas:

Observación: es el instrumento que a lo largo del proceso permitió hacer una exploración del contexto y del ambiente en el cual se estuvo desenvolviendo todo el proceso y de las actividades que se desarrollaron secuencialmente, lo que favoreció la comprensión de situaciones que incluso pasaron por el rótulo de lenguaje no verbal, pero que de esta forma contiene los hechos relevantes sucedidos a lo largo de la investigación.

Entrevista: con la aplicación de este instrumento se logró el intercambio de información para que, de primera mano, llegar a comprender el tipo de dificultad presentada a lo largo del proceso, los aciertos durante el mismo, el grado de participación, el nivel de motivación, y la evaluación a pares que hicieron los participantes del estudio.

Documentos: a través de los documentos se recogieron evidencias de los logros de la investigación, ya que se elaboró un cuestionario dirigido para poner de manifiesto si se lograron alcanzar los objetivos trazados, pero igualmente a través de ellos se pidió a los participantes que de manera libre entregaran un material de elaboración propia e independiente, el cual permitió soportar apropiadamente si se pudo alcanzar la dimensión cognitiva de la investigación.

Cabe mencionar que, en la investigación evaluativa, la estrategia metodológica basada en la observación debía poseer un carácter científico (Tejedor, 2000), es decir, tenía que contemplar técnicas que permitiesen el mayor control y objetividad del fenómeno educativo en análisis; y es 
que al hablar del carácter científico de la observación implica que ella debe ser controlada, objetiva y comprobable. Es por ello que para este proceso investigativo se consideró la participación de dos docentes que acompañaron la etapa de observación, y así tener otras perspectivas de estudio, de evitar sesgos personales y de confirmar datos que puedan resultar del proceso de entrevistas o de situaciones delicadas (Hernández, Fernández \& Baptista, 2010).

Ahora bien, para fundamentar como es debido la utilización de los instrumentos escogidos, es importante anotar que en el registro de observación de conductas en el campo, Salkind (1999) menciona cuatro categorías para la aplicación del registro: registro de duración en el cual el observador utiliza dispositivos para tomar tiempo y medir una conducta determinada; registro de frecuencia donde se toma nota de la recurrencia de determinada conducta; registro de intervalo en donde se observa la actuación de alguien durante determinado tiempo; finalmente se tiene el registro continuo, en donde se siguen todas las conductas de la persona sin importar la especificidad de su contenido. En la aplicación de este instrumento, se tuvo contemplado llevar registros de manera abierta a manera de bitácora, para luego hacer un registro sobre aquellos aspectos que se consideren más relevantes.

En lo concerniente a la aplicación de las entrevistas, al igual que en el caso anterior se requirió de un plan previo, bien detallado, el cual permitió realizar las preguntas adecuadas en el momento justo (Stake, 1999); para ello es necesario tener presente en todo momento que el objetivo de la entrevista debe ser capturar la mayor información posible de situaciones y experiencias del entrevistado, de tal forma que ellas aporten a la pregunta de investigación y estén en línea con los objetivos trazados, por lo que se debe crear un clima da favorabilidad, con un lenguaje acorde a la situación y en el que haya empatía, desarrollando un nivel acorde de escucha activa.

Sin embargo, como toda herramienta utilizada para la recolección de datos, también contiene be- neficios y limitaciones; los beneficios y la riqueza derivada de su aplicación radica en la confluencia de los significados y de las diversas interpretaciones que cada persona hace de la experiencia de participar en el proceso investigativo, y su limitación se deriva del mismo hecho, ya que al tener un carácter único y de relevancia personal no puede afirmar el descubrimiento de una generalidad; con todo y eso, la entrevista se constituye en un elemento que privilegia el intercambio de información y que aporta significado a la investigación educativa (Peón, 2004).

En cuanto al diseño de la entrevista, se contempló una entrevista semiestructurada y preguntas de tipo abierto, iniciando con este último tipo de entrevista, y en la medida del avance del trabajo de campo poder llegar a la entrevista, con un guión previamente elaborado, pero siempre dejando campo para que los participantes se expresaran de forma abierta y pudieran aportar elementos valiosos que contribuyeran a la elaboración de conclusiones significativas en el proceso investigativo.

El último de los instrumentos utilizado hacer referencia al análisis documental; para seleccionar los documentos que den validez a la información, se tuvo especial cuidado en elegir aquellos que verdaderamente pudiesen ser reveladores y pudiesen proporcionar información de valor que estuviese relacionada con el planteamiento del problema (Hernández, Fernández \& Baptista, 2010). Para el presente estudio se diseñó un cuestionario con preguntas dirigidas con las cuales se pretendía tener información acerca del grado de comprensión y asimilación de los temas, y al final del estudio se proyectó recoger un escrito con preguntas abiertas, en las cuales se pudiera corroborar la información obtenida en los procesos de observación y entrevista.

\section{Aplicación de instrumentos}

Para aplicar los instrumentos anteriormente mencionados en el curso de la investigación, se contó 
con un diseño muy flexible, ya que existía la probabilidad que, dado el carácter de la investigación, pudieran aparecer algunos resultados que le entregaran nuevas posibilidades al estudio. En vista de lo anterior, se tuvo planteado llevar un registro detallado de las observaciones obtenidas, las entrevistas realizadas y los análisis documentales practicados.

El procedimiento para la aplicación de los instrumentos se diseñó con base en los cuatro componentes descritos por Rodríguez (1996) como lo son la fase preparatoria, el trabajo de campo, la fase analítica y la fase informativa. La fase de preparación se inició desde el mismo momento en que surge la inquietud para vincular medios tecnológicos en un ambiente pedagógico en donde existe la certeza de que el recurso educativo tecnológico es muy válido, pero que también procura dar un cobijo para que los estudiantes no se esquematicen en la sobreutilización de los dispositivos electrónicos, entendiendo que existe una fundamentación antropológica en la educación Waldorf; es así como se establece un diseño que permita la integración entre los recursos de última tecnología disponibles en la red y los contenidos curriculares, para lo cual se hace una primera exploración tanto de los recursos educativos abiertos como del plan de aula conformado para el año lectivo, y que está conforme al contenido del currículo; estas etapas se materializan en el marco teórico del proyecto de investigación.

Para ello proceso de ejecución del trabajo de campo se tuvo previsto realizarlo de manera paulatina y progresiva, en donde el contacto inicial presupone un acercamiento informal, con el propósito de familiarizar a los participantes con el entorno en el cual se va a interactuar, para acercarlos ya con carácter formal a un espacio y a unas condiciones de trabajo óptimas, en las que cada participante asuma el papel esperado y en las que se vayan aplicando los instrumentos de recolección de los datos que proporcionaran la información requerida.

Para la recolección de la información del trabajo en campo, se hizo uso de recursos como fotogra- fías, observaciones estructuradas y no estructuradas, cuestionarios aplicados, entrevistas y análisis de documentos de apoyo, con lo que se buscó involucrarse en el ambiente de la investigación, capturar la información que se considere valiosa, y así focalizarse en los aspectos más relevantes del estudio. Para las observaciones se dispuso llevar una bitácora de análisis personal; dentro de los documentos oficiales, se contempló el plan de aula y el currículo, ya que ellos albergan, tanto de forma amplia como de forma específica, los contenidos del área, los que finalmente deben estar en concordancia con los contenidos desarrollados en el campo virtual.

Es importante establecer que los aspectos de relevancia del estudio deban ser lo suficientemente susceptibles de ser identificados, es decir, que deban aparecer en forma continua, para entregar los datos adecuados a la pregunta de investigación, lo cual se le denomina suficiencia y adecuación (Stake, 1999). Solo así se puede generar la validez y confiabilidad que requiere el proceso.

Para la etapa siguiente, la fase analítica, se contempló iniciarla desde el mismo trabajo de campo, ya que a través de la aparición continúa de datos con los diferentes instrumentos, se puede avanzar en la construcción e integración de información, sin llegar con ello a descartar aspectos que se registren de manera esporádica o puntual, pues ellos también se constituyen en aportes valiosos: se debe recordar que con el estudio no se pretende llegar a generalizaciones.

En la fase final de la aplicación de los instrumentos se culmina con la presentación de los informes, en donde básicamente se ofrecen los principales hallazgos, resultados, conclusiones y recomendaciones acerca del estudio realizado; para ello se elaboró un resumen que recogiera de manera sintética todos los datos encontrados, sustentado con tablas, gráficos y soportes, y en donde se pudiera verificar la relación encontrada entre las variables planteadas en la pregunta de investigación. 


\section{Estrategias para el análisis de datos}

Cuando se da inicio al análisis de la información recolectada, como punto de partida debe tratar de confrontarse la relación que se encuentra entre los objetivos y los supuestos del estudio, y verificar si esta realidad es consistente y acorde con la pregunta de investigación (Tamayo, 2005). De acuerdo con Sandoval (2002), existen unas características particulares del diseño cualitativo que se convierten en elementos a considerar al momento mismo, en estrategias que definen la forma como se pueden analizar los datos, ya que el procesamiento de la información depende en alto grado de la simultaneidad de estos rasgos. Las características de las cuales se hace referencia son las siguientes: apertura, que consiste en la posibilidad de incluir nuevos elementos en el diseño investigativo, en función de los resultados encontrados en el proceso mismo de recolección; flexibilidad, que se refiere a la posibilidad de modificar el volumen, la calidad, y los medios para obtener la información; sensibilidad estratégica, que posibilita la modificación en el diseño inicial de acuerdo con las características halladas al momento del contacto efectivo con la dinámica que ofrece la realidad del estudio; y referencialidad, que abarca todas las características anteriores, y que le da un carácter de multiciclo a las fases de la investigación cualitativa, en donde existe la simultaneidad de ellas.

La aplicación de estrategias para el análisis de la información entonces se convierte en el elemento que aterriza a los datos, los orienta en la dirección de los objetivos trazados, para dar una respuesta a la pregunta de investigación; lo cual implica que ellas son el origen para la fiabilidad y validez de la información derivada; la fiabilidad hacer referencia a que el procedimiento utilizado y sus conclusiones pueden dar resultados similares en otros eventos de investigación, mientras que la validez implica que la dirección escogida efectivamente conduce hacia lo que se está buscando (Plaza, 2009).
Tomando en cuenta lo anterior, la técnica de observación que se aplicó en el presente estudio, tuvo como propósito recolectar datos para describir los ambientes de aprendizaje de un modo activo; lo anterior significa que va a intervenir en la estructuración del ámbito de observación como una forma de obtener respuestas a los objetivos y necesidades del estudio. Es decir, se obtuvieron datos del comportamiento de los participantes por medio del contacto directo y en el curso de situaciones específicas, ya que se considera como la técnica e instrumento más utilizado en el análisis de la vida social de los grupos humanos (Albert, 2007).

En la organización y procesamiento de datos provenientes de la investigación, se pudo extraer a grosso modo, aquellos hallazgos que realmente tienen relevancia en cuanto a los objetivos del trabajo investigativo, y que dieran repuesta a la pregunta de investigación. Lo que seguidamente se realiza consiste en la clasificación de la información de acuerdo con las fuentes de donde provienen, lo que permite que en la medida en que se recolecta la información se utilice el criterio de triangulación aplicado a cada fuente, para alcanzar el logro de constructos reales y útiles derivados desde puntos de observación independientes (Stake, 1999).

En el proceso de la triangulación de datos, una vez se hayan efectuado la categorización de la que se habló en las fases de la observación, y el análisis de entrevistas y cuestionarios, se procede a determinar cuáles son los elementos de coincidencia que surgen a partir de las diferentes apreciaciones obtenidas a lo largo del trabajo de campo; para ello se hará utilización del formato "Triangulación de medios". El uso del formato anterior se ha diseñado con base en las características contempladas en la naturaleza del método cualitativo, según las cuales, más que ajustarse a las normas y procedimientos establecidos sobre la manera como hay que analizar datos, es el mismo investigador quien construye su propio análisis (Hernández, Fernández \& Baptista, 2010). 
Finalmente se realiza el proceso de transferencia, que no significa la generalización de los resultados a otras poblaciones, sino que implica que la esencia del resultado del estudio pueda ser aplicada en otros contextos, siempre y cuando la descripción de los ambientes de investigación, los instrumentos de aplicación, los participantes y momentos del estudio sea lo más precisa y amplia posible, para que la utilización del estudio genere la validez de la cual se habló anteriormente.

Para las distintas áreas del conocimiento, los alumnos de la institución no llevan ningún texto guía, sino que todo se hace a través las explicaciones que realiza el docente del área, y los alumnos deben consignar sus apuntes en un cuaderno estándar para todos los grados, el cual está diseñado sin ningún tipo de línea o de cuadrícula, como requerimiento establecido dentro de los lineamientos de la pedagogía; el hecho de no tener textos guía permite que los alumnos puedan acceder a distintas fuente de consulta para enriquecer sus actividades de aprendizaje.

En cuanto al proceso de plasmar todas las observaciones realizadas, se llevó una bitácora de trabajo en la cual se hacía el registro de todas las conductas observables y observadas en cada una de las sesiones de trabajo, sin tratar de caer en el exceso de detalles, que en algún momento del estudio pudiesen llegar a producir sesgos o registros carentes de valor; la función de la bitácora es documentar el procedimiento empleado a lo largo del proceso de observación, por lo cual se constituye en un elemento de credibilidad para el trabajo realizado; posteriormente las conductas observadas y registradas en bitácora se plasmaron en tablas siguiendo las categorías mencionadas por Salkind (1999) para la aplicación del instrumento de la observación.

\section{Planteamientos y conclusiones}

Las tecnologías de la información y la comunicación se han convertido en una interesante oportu- nidad para innovar dentro de los distintos esquemas educativos, pero a su vez han pasado a ser todo un desafío en la gestión del aprendizaje, ya que el docente debe asumir su activación como un instrumento didáctico a gran escala, procurando que ellas se puedan involucrar como un elemento imprescindible a considerar dentro del marco de las nuevas políticas educativas (Hepp, 2008); y es que las TIC han traído consigo una variedad de recursos educativos tecnológicos a gran escala, de amplia disponibilidad, lo cual ha permitido la transformación de las prácticas educativas durante este último tiempo, mediante estrategias que permitan orientar a los estudiantes para la construcción de aprendizajes significativos.

El gran reto que se planteó con el presente proyecto de investigación pretendía establecer la manera en que se podría facilitar la adquisición del conocimiento de determinados contenidos matemáticos a partir de dos variables: la utilización de recursos educativos abiertos y su influencia en el proceso de enseñanza-aprendizaje. Al tratar de encontrar las evidencias en el estudio desarrollado, se buscaba determinar cuál es el impacto producido para el desarrollo de las competencias apoyadas por la utilización de las tecnologías digitales; y el primer hallazgo a mencionar parte del hecho de saber que la accesibilidad a las plataformas constituyó un gran aliciente para el estudiante, no solo desde la facilidad del acceso mismo, sino desde la posibilidad de contar con un recurso didáctico de manera permanente, ágil, novedoso y de alguna manera, divertido para ellos.

Al analizar la incidencia de este recurso en el proceso de construcción del conocimiento en estudiantes de una pedagogía para nada proclive al uso de herramientas de índole tecnológica, se puede llegar a establecer que siempre el proceso educativo estará favorecido con la inclusión de elementos que representen la posibilidad de nuevas tendencias al interior del aula. Sin embargo, como lo anotó uno de los mismos estudiantes en el cuestionario aplicado, tampoco se puede garantizar que la vinculación de la tecnología se convierta en garantía de aprendizaje. 
En este sentido, la categoría de motivación encontrada a través de la triangulación de los instrumentos permite llegar a la conclusión que los recursos educativos abiertos constituyen un detonante importante para el desarrollo y fortalecimiento de competencias de manera autónoma, pues son los mismos estudiantes que se convierten en gestores, promoviendo una mejor calidad educativa, ya que la labor del docente se centra en facilitar fases de construcción de aprendizajes de significación, al direccionar actividades, y convertirse en un guía durante todo el proceso.

$\mathrm{Y}$ es que dentro de los lineamientos estipulados por el Ministerio de Educación Nacional en el programa "Colombia Aprende" (2014), se ha contemplado valorar la pertinencia de nuevos actores en el campo educativo, de acuerdo con los requerimientos identificados dentro de los lineamientos de la estrategia nacional para la mejora de la educación; visto así, en el desarrollo del trabajo se pudo establecer que hubo propósito, coherencia y conveniencia con respecto al desarrollo de los temas y su tratamiento en cuanto a los documentos oficiales como el currículo y el plan de aula, lo que permite hablar de la pertinencia en el trabajo de los contenidos aplicados, así como en el proceso de investigación..

Al hablar de pertinencia en cuanto al empleo de los recursos abiertos durante el proceso investigativo, se puede determinar fundamentalmente a partir de dos categorías mencionadas en el capítulo anterior: la utilización del tiempo y la evaluación. Por una parte, se encontró que el tiempo, cualquiera sea la dimensión en que se quiera asumir, fue utilizado provechosamente por los actores del estudio, encontrando un alto grado de satisfacción entre ellos porque el recurso dejó grandes beneficios como la posibilidad de cumplir con tiempos para el desarrollo del plan de aula, como también la posibilidad de desarrollar actividades académicas y compromisos alternativos de parte de los estudiantes. De otra parte, en cuanto a la categoría de evaluación, se hizo evidente el hallazgo de cambios significativos detectados en los alumnos: se puede hablar de desarrollo de competencias para la autogestión, logro de los objetivos de aprendizaje, equidad en los criterios establecidos dentro de la rúbrica, fortalecimiento de habilidades para desarrollar trabajo colaborativo.

Las categorías que se pudieron establecer derivadas del estudio, a saber: motivación, accesibilidad, compromiso escolar, evaluación, resultados académicos, utilización del tiempo, y calidad del proceso, permiten emitir en forma puntual las siguientes conclusiones:

- La utilización de los recursos educativos abiertos permite el desarrollo y fortalecimiento de competencias, siempre que exista una adecuada planificación y coherencia con el currículo escolar.

- Las competencias desarrolladas tienen un alcance superior a la asimilación de contenidos académicos, ya que se alcanzaron a detectar la conformación de competencias para la vida: procesos de autogestión, aprendizajes colaborativos, contenidos con carácter de transversalidad.

- La integración de elementos de innovación tecnológica al interior del aula, conlleva a que los alumnos se sientan atraídos hacia los temas, y logren motivarse hacia el logro de aprendizajes de significado.

- La herramienta tecnológica por sí sola no garantiza el aprendizaje.

- La innovación como tal, debidamente fundamentada y explicada en sus alcances y objetivos, permite mayor sentido de pertenencia y compromiso con los procesos relacionados con el aprendizaje.

- Los ambientes de enseñanza altamente enriquecidos con recursos didácticos novedosos, conducen a un mayor afianzamiento de los contenidos y al alcance de mejores niveles calidad de los procesos de aprendizaje.

Con base en los puntos anteriores, y como una gran conclusión general al proceso desarrollado, se puede afirmar que los recursos educativos 
abiertos se pueden convertir en una herramienta valiosa para fortalecer competencias y consolidar los aprendizajes de forma significativa para los alumnos, pero definitivamente ellos deben ser planificados en forma metódica, de tal forma que efectivamente puedan facilitar el rol del docente y que sirvan para establecer nuevas formas de interacción tanto académicas como sociales.

\section{Recomendaciones}

Dada la creciente demanda que se tiene acerca del uso de R.E.A. en distintos niveles del sector educativo, es preciso que se haga una planificación consciente y lo mayor detallada posible acerca de cuáles son las formas en que ellas se van a involucrar al interior del aula, y en donde se contemplen desde los repositorios a incluir, así como los momentos en que se considere apropiada su vinculación para la construcción del aprendizaje.

Derivado de lo anterior, es preciso que el docente facilitador cuente con un proceso de capacitación previo, en el cual se prepare no solo desde el aspecto tecnológico sino desde los mismos contenidos que se ofrezcan en la o las plataformas escogidas. Este es un hecho que permite flexibilizar los procesos, pues en ciertos momentos del trabajo, se debe contar con la posibilidad de vincular nuevos elementos que mantengan el carácter de innovación que se le quiere dar.

Y es que cuando se cuenta con una capacitación apropiada y la experiencia necesaria para el manejo de los recursos tecnológicos, se puede llegar a profundizar mucho más en algunos otros aspectos del trabajo investigativo, ya que se le puede permitir a los propios alumnos participar, en forma guiada y con criterios claros, del diseño de las actividades de aprendizaje, e incluso vincularlos en forma más asertiva para que diseñen los instrumentos y rúbricas con que ellos mismos serán evaluados. Vale la pena recordar que uno de los hallazgos valiosos del estudio se encuentra en el aprendizaje colaborativo que los estudiantes pu- dieron desarrollar, el cual se hizo evidente en sus mismas manifestaciones y en la evaluación escrita como finalización del proceso.

Otro aspecto que se recomienda para próximas investigaciones con enfoques metodológicos de carácter cualitativo, es la de mantener los registros de observación de manera desaprensiva; al inicio de la investigación no se tenía contemplada la elaboración de una tabla con distintas categorías, pero fue en el transcurrir de la observación como se fue decantando cada uno de estos aspectos, hasta llegar al punto de convertirse en la base de los hallazgos y para la elaboración de las conclusiones, no importando si las observaciones realizadas se hicieron de manera participativas o no participativas dentro de todo el proceso.

Finalmente, y para contar con un buen indicador acerca de la influencia de los recursos educativos abiertos como herramienta facilitadora del proceso de aprendizaje, para un próximo proceso investigativo, se puede pensar en diseñar y aplicar un instrumento que permita elaborar un dictamen acerca del conjunto y del grado de saberes previos con que llegan los estudiantes al proyecto de investigación, y hacia el final del proceso establecer que tanto se ha modificado este registro inicial cuando se ha hecho uso de repositorios y/o plataformas tecnológicas. Si el docente cuenta con registros históricos observados en los que se haya aplicado la clase magistral, puede acercarse con un alto grado de confiabilidad a determinar que tanto influyen los recursos abiertos educativos en el aprendizaje de los estudiantes. 


\section{Referencias}

Albert, M. (2007). La investigación educativa. Claves teóricas. Madrid: Mc Graw Hill Education.

Área, M. (2008). Innovación pedagógica con TIC y el desarrollo de las competencias informacionales y digitales. Revista Investigación en la escuela, (64). Recuperado de: http://manarea. webs.ull.es/articulos/art16_investigacionescuela.pdf

Arma, M. (2012). El E-learning y las competencias digitales. Revista de Educación y Derecho, (5), Recuperado de: http://revistes.ub.edu/index. php/RED/article/viewFile/3087/3615

Artigue, M (1995). Ingeniería didáctica en educación matemática. Bogotá D.C.: Grupo Editorial Iberoamericano.

Breci, A. \& Villaraga D. (2012). Didáctica de las matemáticas. Documento de apoyo. Cali: Universidad Santo Tomás.

Cervantes, G. (2012). Impacto de los recursos educativos abiertos para el desarrollo de las competencias matemáticas en el nivel de educación básica. Tesis de Maestría. Monterrey: Instituto Tecnológico de Monterrey.

De Guzmán, M. (2006). Enseñanza de las ciencias y la matemática. Revista Iberoamericana de Educación, (43). Recuperado de: https://rieoei. org/historico/documentos/rie43a02.pdf

Delors, J. (1996). La educación encierra un tesoro. Madrid: Ediciones Unesco.

Díaz Barriga F. \& Hernández G. (2002). Estrategias docentes para un aprendizaje significativo. Una interpretación constructivista. $2^{\mathrm{a}}$ Edición. México D.F.: Editorial Mc Graw Hill.
Fernández, J. (2006). Fundamentos de la matemática y circunstancias de su educación. Madrid: Dirección General de Ordenación Académica.

Ferro, C.; Martínez, A. \& Otero, M. (2009). Ventajas del uso de las TIC en el proceso de enseñanza-aprendizaje desde la óptica de los docentes universitarios españoles. Revista Electrónica de Tecnología Educativa, Edutec, (29). Recuperado de: http:/edutec.rediris.es/Revelec2/Revelec 29/artículosn29pdf/5edutec

Flores, M. \& Valenzuela, R. (2011) Fundamentos de investigación educativa Vol. 2. México: Editorial Digital del Tecnológico de Monterrey.

García B. \& Loredo J. (2008). Análisis de la práctica educativa de los docentes: pensamiento, interacción y reflexión. Revista Electrónica de Investigación Educativa. (10). Recuperado de: http://www.scielo.org.mx/scielo.php?pi$\mathrm{d}=$ S1607-40412008000300006\&script $=$ sci_arttext

Gardner, H. (1995). Inteligencias múltiples. Barcelona: Editorial Paidós.

Godino, J. (2003) Fundamentos de la enseñanza y el aprendizaje de las matemáticas para maestros. Granada: Universidad de Granada.

Goleman, D. (1998). La inteligencia emocional. Barcelona: Editorial Kairós.

Hepp, P. (2008). El desafío de las TIC como instrumento de aprendizaje. Las TIC: del aula a la agenda política. Recuperado de : http://www. oei.es/pdfs/las_tic_aula_agenda_politica.pdf

Heinze, A. (2004). Reflections on the Use of Blended Learning. Education in a Changing Environment. Salford: University of Salford.

Hernández R., Fernández C. \& Baptista P. (2010). Metodología de la investigación. México D.F.: Editorial Mc Graw Hill Interamericana. 
Herrington, J. (2000). Moving from an Instructivist to a Constructivist. Multimedia Learning Environment. Journal of Educational Multimedia and Hypermedia, 9(3), Recuperado de: http:// www.editlib.org/p/8084/

Jiménez, F. (2000). Investigación y actividad educativa: una concreción a la diversidad social y cultural. Profesorado, revista de currículum y formación del profesorado, Vol .4 No 1. Universidad Complutense de Madrid. Recuperado de: http://telematica.politicas.unam.mx/biblioteca/archivos/040107021.pdf.

Llinás, R. (2003) El cerebro y el mito del yo. Bogotá: Editorial Norma.

Lozano A. (2011). El éxito en la enseñanza: aspectos didácticos de las facetas del profesor. México D.F.: Editorial Trillas.

Lozano A. \& Herrera, J. (2013). Diseño de programas educativos basados en competencias E.book, México: Instituto Tecnológico y de Estudios Superiores de Monterrey.

Martínez, M. (2000). La investigación cualitativa etnográfica en educación. Manual teórico-práctico. $3^{\text {a }}$ Edición. México D.F.: Editorial Trillas.

Mc Geal, R. (2011). How to build open educational resources. Revista La Educación, (146). Recuperado de: http://www.educoea.org/portal/ La_Educacion_Digital/146/index.html

Ministerio de Educación Nacional de Colombia (2014). Evaluación Educativa. Recuperado de: www.Mineducación.gov.co/1621/w-3-article-179264.html.

Ministerio de Educación Nacional de Colombia (2006). Lineamientos en TIC. Pacto Social por la Educación. Plan Decenal de educación 20062016. Bogotá D.C.

Ministerio de Educación Nacional de Colombia (2006) Programa Colombia Aprende. Bogotá D.C.
Recuperado de : http://www.colombiaaprende.edu.co/html/home/1592/w3

Ministerio Nacional de las T.I.C. de Colombia (2012) Plan Vive Digital Bogotá D.C. Recuperado de : http://www.colombiaaprende.edu.co/ html/home/1592/w3-channel

Ortega, J. (2011). Recursos educativos abiertos para la enseñanza de las matemáticas en ambientes de educación básica enriquecidos con tecnología educativa. Tesis de maestría. Monterrey: Instituto Tecnológico de Monterrey.

Páez, M. R. (2007). El uso del tiempo en la práctica pedagógica de las escuelas adscritas a la Alcaldía Metropolitana. Sapiens: Revista Universitaria de Investigación, 8(2). Recuperado de: http:// www2.scielo.org.ve/scielo.php?script=sci_arttext\&pid=S1317-58152007000200006\&ln$\mathrm{g}=\mathrm{es} \& \mathrm{nrm}=\mathrm{i}$

Peón, F. (2004). Un acto metodológico básico de la investigación social: la entrevista cualitativa. $\mathrm{Ob}$ servar, escuchar y comprender sobre la tradición cualitativa en la investigación social. México D.F., Flacso. Recuperado de: http://www.textosdigitales.com.ar/ CP/CICLO_BASICO/3.017_

Quintanilla, M. (1981). Tecnología, un enfoque filosófico. Madrid: Fundesco.

Rendón, V. (2012). La computadora llega al aula: La incorporación de tecnologías digitales a la práctica docente. México D.F.: Centro de investigación y de estudios avanzados del Instituto Politécnico Nacional -Sede sur -Departamento de Investigaciones Educativas. Recuperado de: http://www.lets.cinvestav.mx/Portals/0/SiteDocs/TesisSS/Maestria/lets_sur_tesis

Rodríguez, G., García, E. \& Gil, J. (1996). Metodología de la investigación cualitativa. Granada: Ediciones Aljibe.

Salkind, N. (1997). Métodos de investigación. México D.F.: Pearson Educación . 
Sandoval, C. (2002). Investigación cualitativa. Bogotá: Arfo Editores.

Serrano, J. (2010). Uso de software libre para el desarrollo de contenidos educativos. Revista Formación Universitaria, 3(6). Recuperado de: http://www.scielo.cl/scielo.php?pi$\mathrm{d}=$ S0718-50062010000600006\&script $=$ sci

Stake, R. (1999). Investigación con estudio de casos, $2^{\mathrm{a}}$ Edición. Madrid: Ediciones Morata.

Stephenson, J. (2011). Modelos pedagógicos y e-learning. Universitat Oberta de Catalunya. Recuperado de: http://www.iupuebla.com/Maestrias/ MTE/ATLIXCO/MAT_APOYO/Modelos\%20 pedagogicos\%20y\%20e-learning_1-29_91-105. pdf

Tapia, J. (1995). Motivación y aprendizaje en el aula-como enseñar a pensar, Madrid: Aula XXI / Santillana.

Tamayo, M. (2005). Investigación para niños y jóvenes. México D.F.: Editorial Limusa-Grupo Noriega Editor.

Tejedor, F. (2000). El diseño y los diseños en la evaluación de programas. Revista de Investigación Educativa, 18(2). Recuperado de: http://revistas.um.es/rie/article/viewFile/121021/113711 